\title{
Pronóstico difuso del examen general de egreso de licenciatura para ingeniería en computación de la Universidad Autónoma del Estado de México
}

\author{
Sandra Silvia Roblero Aguilar, Héctor Rafael Orozco Aguirre \\ Universidad Autónoma del Estado de México, \\ Centro Universitario UAEM Valle de México, \\ Atizapán de Zaragoza, Estado de México, México \\ ssrauaemex@hotmail.com, hrorozcoa@uaemex.mx
}

\begin{abstract}
Resumen. Este artículo tiene como finalidad dar a conocer un mecanismo computacional, que permite generar un pronóstico utilizando lógica difusa, para los pasantes de la carrera de Ingeniería en Computación de la Universidad Autónoma del Estado de México (UAEM), a través de la opción de titulación por el Examen General de Egreso de Licenciatura (EGEL) aplicado por el Centro Nacional de Evaluación para la Educación Superior (CENEVAL).
\end{abstract}

Palabras clave: lógica difusa, pronóstico, examen general de egreso de licenciatura (EGEL), eficiencia terminal, eficiencia de titulación, UAEM.

\section{Introducción}

Actualmente, en México la Eficiencia Terminal (ET) es el principal indicador empleado por el Sistema Educativo Nacional con el objetivo de evaluar la labor de formación profesional de cualquier Institución Educativa (IE). La Dirección General de Planeación y Estadística Educativa de la Secretaría de Educación Pública (SEP) la define como: "la relación porcentual entre los egresados de un nivel educativo dado y el número de estudiantes que ingresaron al primer grado de este nivel educativo $\mathrm{n}$ años antes" [1], que matemáticamente se expresa así:

$$
\operatorname{ET}(\mathrm{n})=(\operatorname{Egresos}(\mathrm{n}) / \text { Nuevo Ingreso }(\mathrm{n})) * 100
$$

Con la finalidad de que exista una evaluación real de las Instituciones de Educación Superior (IES), es necesario tomar en cuenta aparte de la ET, el número de pasantes que logran graduarse, es decir, aquellos que impactan positivamente en el índice de la Eficiencia de Titulación (ETi), que está dada por la expresión:

$$
\operatorname{ETi}(\mathrm{n})=(\operatorname{Titulados}(\mathrm{n}) / \text { Nuevo Ingreso(n) }) * 100
$$

Dado lo anterior, para poder incrementar la ETi, las IES consideran como válidas a distintas modalidades en sus procesos de titulación, en estas se incluyen: aprovecha- 
miento académico, créditos de estudios avanzados, el Examen General de Egreso de Licenciatura (EGEL), entre otras, como lo son los trabajos escritos.

Al hacer un análisis de los resultados presentados en el anuario estadístico de la Asociación Nacional de Universidades e Instituciones de Educación Superior (ANUIES), correspondiente al ciclo escolar 2012-2013, que es el último reportado hasta noviembre de 2014 [2], y de la Agenda Estadística de la Universidad Autónoma del Estado de México (UAEM) para el mismo ciclo escolar [3], se tiene la siguiente tabla comparativa:

Tabla 1. Comparativo de indicadores de la ET y la ETi del ciclo escolar 2012-2013

\begin{tabular}{|c|c|c|c|c|c|c|}
\cline { 2 - 7 } \multicolumn{1}{c|}{} & Matricula & $\begin{array}{c}\text { Nuevo } \\
\text { Ingreso }\end{array}$ & Egresados & $\begin{array}{c}\text { Eficiencia } \\
\text { Terminal }\end{array}$ & Titulación & $\begin{array}{c}\text { Eficiencia } \\
\text { de } \\
\text { Titulación }\end{array}$ \\
\hline Nacional & $\mathbf{3 , 3 0 9 . 2 2 1}$ & $\mathbf{8 7 7 , 4 7 6}$ & $\mathbf{4 6 9 , 5 7 3}$ & $\mathbf{5 3 . 5 \%}$ & $\mathbf{3 4 3 , 6 1 3}$ & $\mathbf{3 9 . 1 \%}$ \\
\hline UAEM & $\mathbf{4 5 , 1 6 1}$ & $\mathbf{1 2 , 2 3 5}$ & $\mathbf{5 , 6 7 4}$ & $\mathbf{4 6 . 4 \%}$ & $\mathbf{4 , 0 5 6}$ & $\mathbf{3 3 . 1 \%}$ \\
\hline $\begin{array}{c}\text { CU UAEM } \\
\text { VM }^{1}\end{array}$ & $\mathbf{3 , 0 5 3}$ & $\mathbf{8 6 4}$ & $\mathbf{3 9 6}$ & $\mathbf{4 5 . 8 \%}$ & $\mathbf{1 8 9}$ & $\mathbf{2 1 . 8 \%}$ \\
\hline ICO $^{2}$ & $\mathbf{2 1 1}$ & $\mathbf{8 4}$ & $\mathbf{1 7}$ & $\mathbf{2 0 . 2 \%}$ & $\mathbf{9}$ & $\mathbf{1 0 . 7 \%}$ \\
\hline
\end{tabular}

Como se puede observar en la tabla anterior, en el CU UAEM VM, la carrera de ICO requiere una atención inmediata, para que se pueda establecer mecanismos que conlleven a incrementar la ETi. Adicionalmente, es importante mencionar que el Reglamento de Evaluación Profesional de la UAEM [4], considera como meta del plan rector la necesidad de mejorar la capacidad profesional de egresados para favorecer su inserción laboral y progreso profesional, fijándose como porcentajes lograr un índice de titulación por cohorte de $23.5 \%$, de los cuales el $29 \%$ de los egresados que se titulan lo hagan a través del EGEL. De acuerdo a esto, si 864 alumnos son de nuevo ingreso en el CU UAEM VM, esto implica que para cumplir con la meta, entonces 203 egresados se deben titular y de estos 59 por EGEL. Para la carrera de ICO, si 84 son de nuevo ingreso, 20 se van a titular y 6 lo harán por el EGEL.

Si se desea impulsar al EGEL como una modalidad de titulación, se tendrán que considerar: los contenidos de los Programas Educativos (PE), las necesidades del campo laboral y que los alumnos adquieran las competencias profesionales a ser evaluadas. De manera tradicional, la preparación para el EGEL no contempla que las áreas y sub-áreas de evaluación hayan sido cubiertas en los contenidos de los PEs. Esto dice, que el anticipar un resultado es hasta cierto punto ciego, ya que no se sabe con exactitud qué Unidades de Aprendizaje (UDAs) corresponden a cada área, cuál es la más fortalecida y las que necesitan ser reforzadas. Para ello, se propone un mecanismo computacional basado en lógica difusa para generar un pronóstico del resultado del EGEL. Esto es para contar con un resultado aproximado que pudiera obtener cualquier sustentante de ICO. Dicho mecanismo, se describirá en la sección 4 de este artículo.

Centro Universitario UAEM Valle de México.

2 Ingeniería en Computación. 


\section{Aspectos generales del EGEL CENEVAL}

\subsection{Descripción}

Como asociación civil sin fines de lucro, el Centro Nacional de Evaluación para la Educación Superior (CENEVAL), tiene la encomienda de diseñar y aplicar instrumentos de evaluación de conocimientos, habilidades y competencias, así como el análisis y la difusión de resultados. Uno de esos instrumentos es el EGEL, el cual consiste en una prueba a nivel nacional, que se especializa por carrera profesional. Esta prueba tiene como objetivo identificar en qué medida los egresados de una licenciatura en específico cuentan con los conocimientos y las habilidades esenciales para el inicio del ejercicio profesional en el campo laboral.

El encargado del diseño, revisión y actualización de cada variante del EGEL, es un Consejo Técnico (CT), que se conforma por representantes de IES públicas y privadas, así como por colegios o asociaciones de profesionales e instancias empleadoras del sector público o privado del país. Cada CT se encarga de validar a una o más licenciaturas en particular, con base en las necesidades de las instancias empleadoras del sector público o privado.

El EGEL en el caso de Ingeniería Computacional [5], es el examen aplicado a ICO de la UAEM, mismo que está conformado por 186 reactivos de opción múltiple con cuatro opciones de respuesta, de las cuales sólo una es la correcta. Este examen comprende las siguientes cinco áreas de conocimiento:

1. Selección de Sistemas Computacionales para Aplicaciones Específicas (SSCAE).

2. Nuevas Tecnologías para la Implementación de Sistemas de Cómputo (NTISC).

3. Desarrollo de Hardware y su Software Asociado para Aplicaciones Específicas (DHSAAE).

4. Adaptación de Hardware y/o Software para Aplicaciones Específicas (AHSAE).

5. Redes de Cómputo para Necesidades Específicas (RCNE).

Dicho examen, también puede ser empleado para evaluar otras carreras, tal es el caso de Ingeniería en Sistemas y Comunicaciones del CU UAEM VM.

\section{2. Índice CENEVAL}

Las calificaciones están expresadas en una escala especial llamada Índice CENEVAL (IC) [5], que va de los 700 puntos, calificación más baja; a los 1300 puntos, calificación más alta. Este índice se utiliza en las pruebas con referencia a criterio, con el objetivo de categorizar el resultado obtenido en alguno de los rangos de puntuación, mismos que serán explicados más adelante.

En lo que respecta a los resultados, se establece una media teórica de 1000 puntos igual a $50 \%$ de aciertos y una desviación estándar de 100 puntos igual a $16.67 \%$. Lo 
anterior debido a que las pruebas se diseñan para tener una distribución de las puntuaciones con esa media y desviación estándar.

\subsection{Rangos de puntuación}

En cada una de las áreas del examen se consideran tres niveles de desempeño: Aún No Satisfactorio (ANS), Satisfactorio (DS) y Sobresaliente (DSS), en los cuales se clasifica a los sustentantes en función del desempeño mostrado, de conformidad con los siguientes rangos de puntuación:

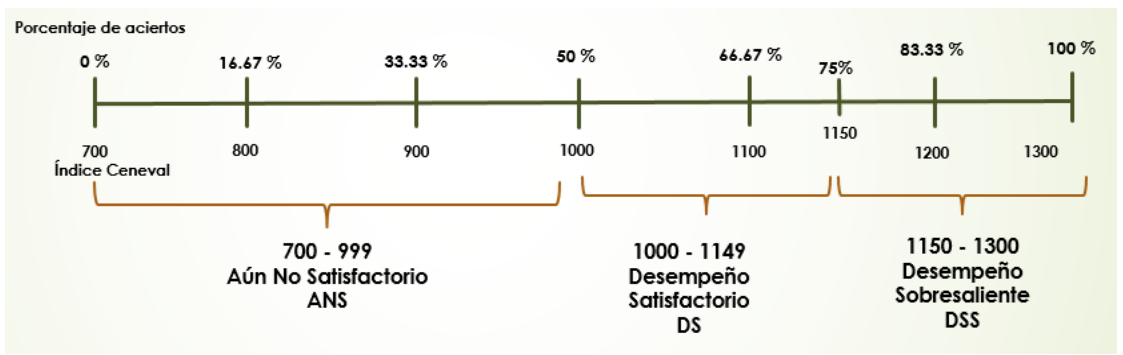

Fig. 1. Distribución de las puntuaciones del EGEL

En la escala de 0-100, el CT fija los cortes que corresponden a los puntajes $1000 \mathrm{y}$ 1150 de IC. Esto hace que independientemente de en qué lugar el CT haya fijado los puntos de corte, el primero de estos siempre es 1000 y el segundo siempre es 1150 . Con lo cual, sin importar el área a la cual se esté haciendo referencia, una calificación superior o igual a 1000 puntos indica un nivel de DS y una superior o igual a 1150, un nivel de DSS. Es decir, en cada una de las áreas del examen, la descripción de los niveles de desempeño permite conocer qué problemas y situaciones es capaz de resolver un sustentante cuando alcanza un DS, y cuáles, cuando alcanza un DSS.

Considerando el nivel de desempeño alcanzado por el sustentante en cada una de las áreas, se determina si éste se hace acreedor a algún Testimonio de Desempeño (TD), con base en los criterios establecidos por el CT, que a continuación se detallan:

- Testimonio de Desempeño Satisfactorio (TDS): al menos cuatro áreas con DS o DSS.

- Testimonio de Desempeño Sobresaliente (TDSS): de las cinco áreas, al menos dos con DSS y las restantes con DS.

Cabe destacar que para este caso, el EGEL está orientado a determinar si los sustentantes son capaces de utilizar lo que han estudiado y aprendido en su licenciatura en situaciones similares a las que se enfrentarán en el ejercicio profesional, así como el hecho de que su contenido se encuentra definido en forma precisa y se validó socialmente. 


\subsection{Resultados del EGEL para el ciclo escolar 2012-2013}

El total de sustentantes que presentaron el EGEL en Ingeniería Computacional durante este ciclo escolar a nivel nacional fue de 3,350 sustentantes [5]. De ellos, 7.8\% (260 sustentantes) obtuvo un TDSS; 37\% (1,240 sustentantes) un TDS; y 55.2\% (1,850 sustentantes) no obtuvo testimonio. De nueva cuenta, en el CU UAEM VM como referencia, para el mismo ciclo, de 238 sustentantes, el $4.62 \%$ (11 sustentantes) alcanzó un TDSS; 48.7\% (116 sustentantes) un TDS; y 46.6\% (111 sustentantes) se quedó sin testimonio. Para la carrera de ICO, los sustentantes que presentaron el EGEL fueron 18 sustentantes. De los cuales, el 11.1\% (2 sustentantes) consiguió un TDSS; el 33.3\% (6 sustentantes) un TDS; y el 55.56\% (10 sustentantes) no obtuvo testimonio.

En la siguiente figura, se observa una gráfica del comparativo de resultados que en términos generales se puede decir, que aproximadamente el 55\% de los sustentantes no alcanza el puntaje mínimo requerido por el EGEL para obtener un TDS y por ende, los sustentantes no pueden titularse por esta modalidad.

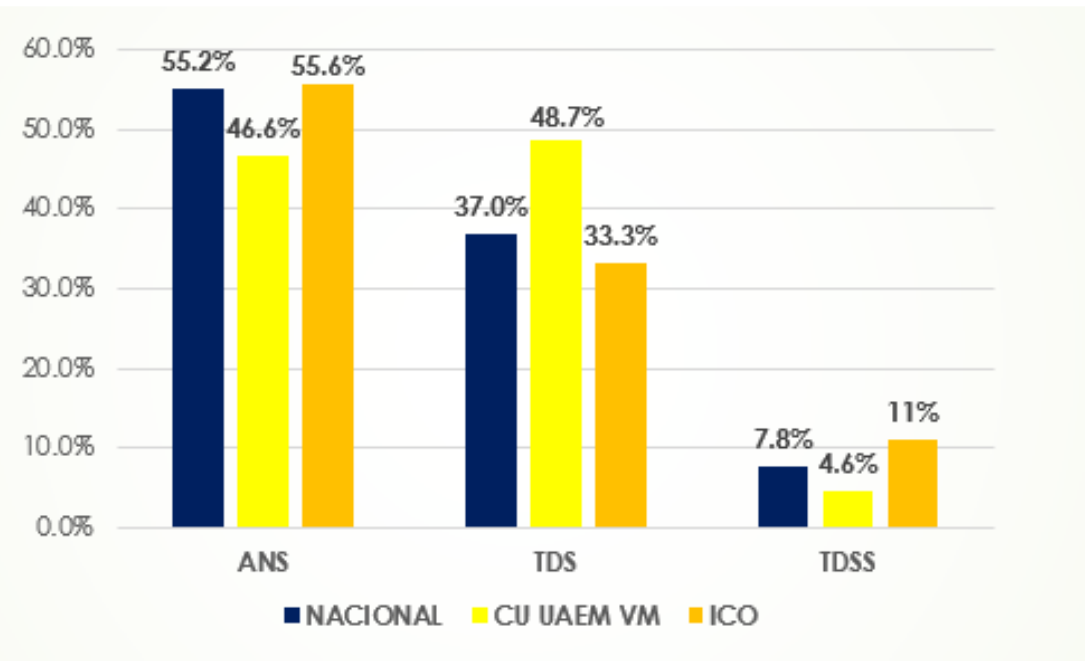

Fig. 2. Comparativo de los resultados del EGEL

\section{Lógica difusa}

\subsection{Panorama general}

El ser humano muestra dificultad para tomar decisiones cuando se tiene información imprecisa. La lógica difusa [6] fue creada para emular la lógica humana y tomar decisiones acertadas a pesar de la poca información disponible. Esta es una herramienta flexible que se basa en reglas lingüísticas dictadas por expertos y que tiene 
como objetivo principal la formalización o mecanización de un sistema lógico para la evaluación y generación de decisiones.

La graduación y granulación (ver fig. 3) forman el núcleo de la lógica difusa, siendo sus principales características distinguibles [7]. Más específicamente, cuando se le emplea, todo dato o información es o se permite que sea graduado, es decir, es una cuestión de grado o, equivalentemente, difuso. Además, de forma similar todo es o se permite por igual que sea granular, un gránulo puede ser un grupo de atributos-valores unidos de manera indistinta, semejante, próxima o funcional. De una manera cualitativa, graduación y granulación juegan papeles fundamentales en la cognición humana.

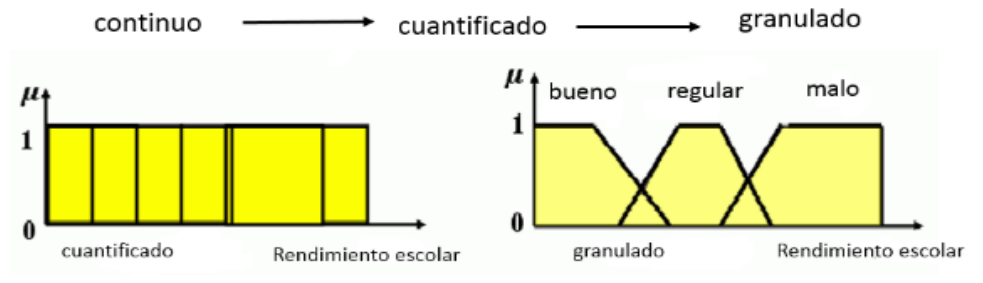

Fig. 3. Granulación y graduación de una variable lingüística

La lógica difusa es [8] un conjunto de principios matemáticos basados en grados de membresía o pertenencia, cuya función es modelar información. Este modelado se hace con base en reglas lingüísticas que aproximan una función mediante la relación de entradas y salidas del sistema. Esta lógica presenta rangos de pertenencia dentro de un intervalo entre 0 y 1 , a diferencia de la lógica convencional, en la que el rango se limita a dos valores: el cero o el uno. La lógica difusa consta de tres etapas para obtener el resultado deseado (ver fig. 4). Estas se explican a continuación:

- Etapa 1: se basa en un proceso donde las variables tienen un grado de incertidumbre metalingüístico. Es decir, el rango de valores de cada variable puede clasificarse por conjuntos difusos, originando el universo del discurso. Con ello, los valores pasan a un proceso de fusificación que los categoriza en un rango de pertenencia entre 0 y 1 que pertenece a un conjunto difuso. Los conjuntos difusos son caracterizados mediante funciones de pertenencia, las cuales están sintonizadas al punto de operación adecuado para el funcionamiento del sistema, es decir, las reglas de inferencia que serán empleadas.

- Etapa 2: se proponen reglas lingüísticas conocidas como de inferencia. Con esto, el grado de pertenencia de cada una de las variables se evalúa en un subconjunto de estas reglas. Cada subconjunto se usa para determinar una consecuencia, es decir, asignar un grado de pertenencia a un conjunto difuso que caracteriza o da las salidas para las variables de entrada.

- Etapa 3: consiste en determinar los valores óptimos de salida, mediante un mecanismo conocido como defusificación, el cual consiste en pasar el grado de perte- 
nencia, proveniente de la consecuencia de la regla de inferencia activada, a un valor nítido o real, es decir, con el fin de obtener un valor cuantificable.

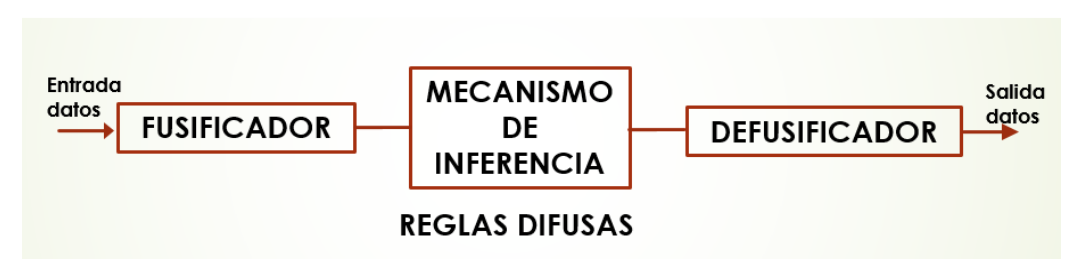

Fig. 4. Esquema general de un mecanismo de inferencia difuso

\subsection{Funciones de pertenencia}

Las funciones de pertenencia son una forma de representar gráficamente un conjunto difuso sobre un universo. De esta manera, la función de pertenencia de un conjunto indica el grado en que cada elemento de un universo dado, pertenece a dicho conjunto. Es decir, la función de pertenencia de un conjunto $A$ sobre un universo $X$ será de la forma: $\mu A: X \rightarrow[0,1]$, donde $\mu A(x)=r$, si $r$ es el grado en que $X$ pertenece al conjunto $A$.

Si el conjunto es nítido, su función de pertenencia (función característica) tomará los valores en $\{0,1\}$, mientras que si es difuso, los tomará en el intervalo [0,1]. Si $\mu A(x)=0$ el elemento no pertenece al conjunto, si $\mu A(x)=1$ el elemento sí pertenece totalmente al conjunto. La función característica del conjunto de los elementos que verifican un predicado clásico está perfectamente determinada. No ocurre lo mismo cuando se intenta obtener la función de pertenencia de un conjunto formado por los elementos que verifican un predicado difuso. Dicha función dependerá del contexto (o universo) en el que se trabaje, del experto, del usuario, de la aplicación a construir, etc.

A la hora de determinar una función de pertenencia, normalmente se eligen funciones sencillas, para que los cálculos no sean complicados. En particular, en aplicaciones en distintos entornos, son muy utilizadas las siguientes:

- Función triangular: definida mediante el límite inferior $a$, el superior $b$ y el valor modal $m$, tal que $a<m<b$. La función no siempre es simétrica.

- Función trapezoidal: definida por sus límites inferior $a$, superior $d$, y los límites de soporte inferior $b$ y superior $c$, tal que $a<b<c<d$. En este caso, si los valores de b y c son iguales, se obtiene una función triangular.

\section{3. ¿Por qué emplear la lógica difusa?}

Principalmente, la lógica difusa está enfocada a la toma de decisiones cuando existen datos o conocimientos inciertos, habiendo bastantes aplicaciones para la vida real y donde se reemplaza al operador humano por un sistema difuso basado en reglas. En este artículo, se eligió a esta debido a que ofrece varias ventajas, descritas como sigue: 
- Al momento de dar un pronóstico, proporciona una manera sencilla y eficaz para extraer conclusiones de vaguedad, ambigua o información imprecisa. Por lo tanto, simula la toma de decisiones humanas y puede trabajar a partir de datos aproximados para obtener soluciones precisas.

- Incorpora una forma alternativa de pensar, lo que permite que todo pronóstico sea modelado con un nivel de abstracción que refleje conocimiento y experiencia a partir de reglas de inferencia.

- Este tipo de lógica permite expresar conocimiento con conceptos subjetivos, tales como los resultados que pueden ser obtenidos en el EGEL: ANS, TDS, TDSS, los cuales pueden ser mapeados de manera exacta dentro de rangos difusos.

- Es un método eficiente que rápidamente proporciona uno o más pronósticos como soluciones.

- Esta ofrece varios beneficios, tales como el rendimiento, simplicidad, bajo costo y productividad en el modelado de un sistema de pronóstico difuso, el cual es explicado en la siguiente sección.

\section{Propuesta de modelo de pronóstico difuso}

\subsection{Análisis}

El plan de estudios de ICO de la UAEM está comprendido de 430 a 450 créditos, de los cuales en el CU UAEM VM se cursan 434. En total, 355 horas son teóricas y 79 son prácticas, repartidas entre 64 UDAs.

El conjunto total de UDAs a partir de este momento será el siguiente universo del discurso $X=$ \{programación estructurada, seguridad en redes, fundamentos de bases de datos, métricas de software, ..., programación paralela y distribuida $\}$ y el predicado $\mathrm{P}=$ "tener membresía para cada una de las áreas correspondientes del EGEL". Se define $\mathrm{P}$ como el subconjunto de UDAs con membresía a dichas áreas.

Por ejemplo, una UDA que sólo pertenece a un área es seguridad en redes, por lo que su función de pertenencia sería:

$\mu_{\mathrm{pSSCAE}}($ seguridad en redes $)=0$;

$\mu_{\mathrm{pNTISC}}($ seguridad en redes $)=0$;

$\mu_{\mathrm{pDHSAAE}}($ seguridad en redes $)=0$;

$\mu_{\mathrm{pAHSAE}}($ seguridad en redes) $=0$;

$\mu_{\mathrm{pRCNE}}($ seguridad en redes $)=1$;

Por otra parte, un caso especial de UDA con función de pertenencia en varias áreas es programación paralela y distribuida:

$\mu_{\mathrm{pSSCAE}}($ programación paralela y distribuida $)=0$;

$\mu_{\mathrm{pNTISC}}($ programación paralela y distribuida) $=0.6$;

$\mu_{\text {pDHSAAE}}$ (programación paralela y distribuida) $=0$;

$\mu_{\text {pAHSAE}}($ programación paralela y distribuida) $=0$;

$\mu_{\mathrm{pRCNE}}($ programación paralela y distribuida $)=0.4$;

En el caso de seguridad en redes, se tiene una membresía con grado 1 en el área RCNE, mientras que programación paralela y distribuida cuenta con un grado de 0.4 
en dicha área, lo cual indica que el $100 \%$ del contenido temático de seguridad en redes corresponde a esta área y sólo el $40 \%$ del contenido temático de programación paralela y distribuida cubre la misma área.

Al concluir la función de pertenencia, se aplica la siguiente ecuación para cada área:

$$
\mathrm{POA}=\sum(\mathrm{PUDA})
$$

Donde POA: pertenencia obtenida por área.

PUDA: ponderación asignada a la UDA para esa área.

Posteriormente, para cada área se calcula un porcentaje de evaluación, considerando las calificaciones obtenidas por el alumno en cada UDA y la evaluación dada al docente de cada UDA, obteniendo la siguiente ecuación:

$$
\mathrm{PA}=(\mathrm{CUDA} * 0.8+\mathrm{CDO} * 0.2) * \mathrm{PUDA} .
$$

Donde PA: es el porcentaje de área.

CUDA: calificación de la UDA.

CDO: calificación del docente.

Como se puede ver, a la calificación obtenida por el alumno en cada UDA se le asignó el $80 \%$ y a la calificación del docente se le otorgó el 20\%. Esta asignación, va en función de que la evaluación docente pudiera no ser objetiva, sin embargo, es un factor a considerar para la apreciación del alumno.

\subsection{Estimaciones}

Con el análisis previo, es posible obtener las siguientes estimaciones:

- Estimación simple: en esta se considera el valor máximo de POA y los rangos de puntuación del IC, tal y como se muestra en la siguiente ecuación:

$$
\mathrm{ES}=\sum(\mathrm{PA}) / \operatorname{Max}(\mathrm{POA}) * 6+700
$$

Donde ES: es la estimación simple por Área.

- Estimación basada en periodos de egreso: aquí se toma como referencia el periodo de egreso del alumno, suponiendo que por cada año de egreso se le resta $0.025 \%$, con ello se tiene la ecuación:

$$
\mathrm{EPE}=\mathrm{ES} *(1-(0.025 * \mathrm{NAEG}))
$$

Donde EPE: es la estimación por periodo de egreso.

NAEG: número de años de egreso.

- Estimación basada en años de experiencia: se considera la experiencia en el campo laboral, por cada año de experiencia se considera un incremento de $0.375 \%$, por lo que la ecuación queda de la siguiente manera:

$$
\mathrm{EAE}=\mathrm{ES} *(1+(0.0375 * \mathrm{NAEX}))
$$


Donde EAE: es la estimación por años de experiencia.

NAEX: número de años de experiencia.

\subsection{Pronóstico}

Considerando las estimaciones anteriores, el siguiente paso es dar un pronóstico del resultado del EGEL. Para ello, se obtiene primero el promedio de las estimaciones descritas anteriormente, aplicando la siguiente ecuación:

$$
\mathrm{PAE}=(\mathrm{ES}+\mathrm{EPE}+\mathrm{EAE}) / 3
$$

Donde PAE: es el pronóstico del área del EGEL.

La ecuación 8 se debe realizar para cada una de las áreas del EGEL.



Fig. 5. Fusificación del área Selección de Sistemas Computacionales para Aplicaciones Específicas

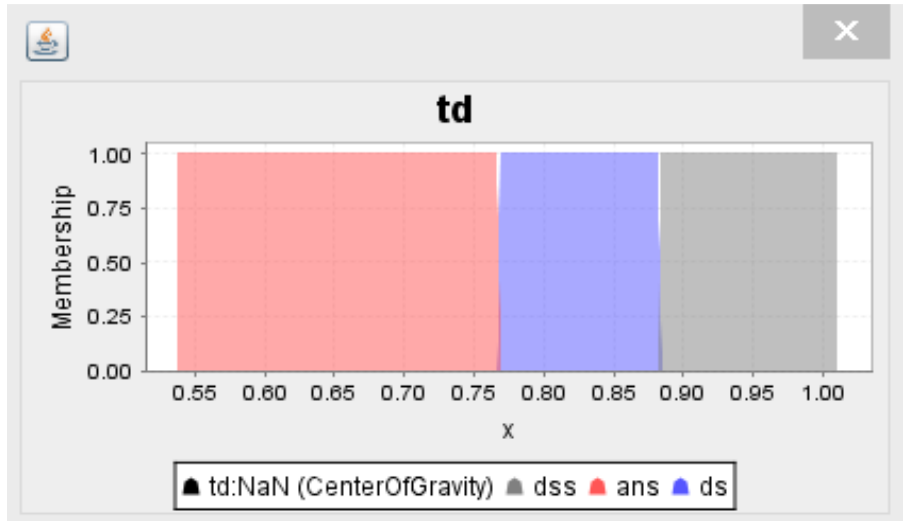

Fig. 6. Fusificación del Testimonio de Desempeño 
La fusificación y defusificación se llevó a cabo empleando jfuzzylogic [9]. A cada una de las áreas de evaluación del EGEL para ICO, se le asignaron los mismos intervalos difusos, ANS, DS y DSS (ver fig. 5 y 6). Como ejemplo, se muestra a continuación lo hecho para el área SSCAE.

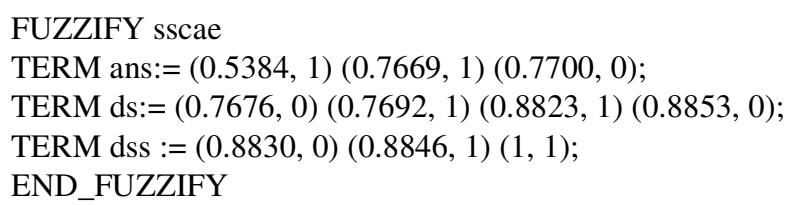

En la defusificación, se necesitan 25 reglas difusas para obtener el TD del pronóstico difuso del resultado del EGEL, de las cuales como muestra se presentan las 5 reglas para obtener el TDSS:

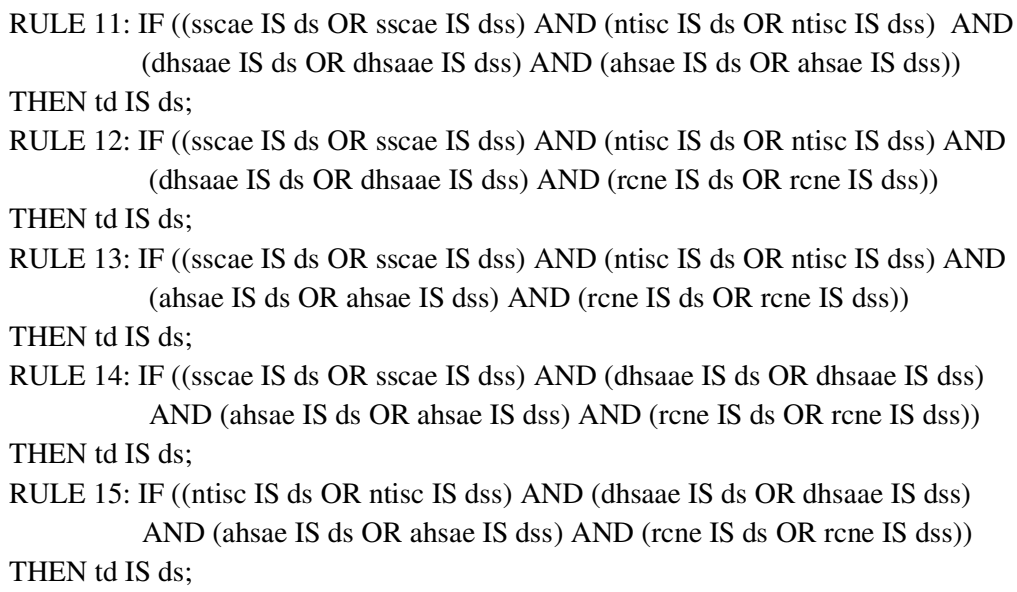

\subsection{Caso de estudio}

En la última aplicación del EGEL, con fecha del 28 de noviembre de 2014, celebrada en el CU UAEM VM, un sustentante elegido al azar obtuvo los siguientes puntajes con un TD dado por un TDS:

SSCAE $=1039$

NTISC $=1134$

DHSAAE $=1070$

AHSAE $=1047$

$\mathrm{RCNE}=1001$

Ahora bien, al aplicar el modelo de pronóstico difuso aquí propuesto, se introdujeron las calificaciones obtenidas por el alumno en cada una de las UDAs y las calificaciones que dicho alumno otorgó a sus profesores en el proceso de apreciación estudiantil, para obtener las estimaciones y el pronóstico descritos anteriormente. 
De acuerdo a las estimaciones mostradas en la fig. 7, se observa que en la carrera de ICO, el área Desarrollo de Hardware y su Software Asociado para Aplicaciones Específicas, es la de mayor presencia y la de menor presencia es el área de Redes de Cómputo para Necesidades Específicas en el plan de estudios, respectivamente. Cabe mencionar, que el EGEL evalúa todas las áreas de manera uniforme y la distribución aquí obtenida no lo es. Dadas las estimaciones obtenidas, el pronóstico para cada una de las áreas es el siguiente:

PAE SSCAE $=1094.1$

PAE NTISC $=1117.1$

PAE DHSAAE $=1146.6$

PAE AHSAE $=1115.2$

PAE RCNE $=976.29$

\begin{tabular}{|c|c|c|c|c|c|c|c|c|}
\hline \multicolumn{9}{|c|}{ INGENIERIA EN COMPUTACION } \\
\hline \multirow[t]{3}{*}{ Alumno: } & - & \multicolumn{3}{|c|}{ No. Cuenta: } & \multicolumn{4}{|l|}{ man } \\
\hline & \multirow{2}{*}{ UNIDAD DE APRENDIZAJE } & \multirow{2}{*}{\multicolumn{2}{|c|}{$\begin{array}{l}\text { CALIFICACIONES } \\
\text { Docente Pasante }\end{array}$}} & $\begin{array}{l}\text { SELECCIÓN DE } \\
\text { SISTEMAS } \\
\text { COMPUTACIONALES }\end{array}$ & $\begin{array}{c}\text { NUEVAS } \\
\text { TECNOLOGIAS } \\
\text { PARA LA }\end{array}$ & $\begin{array}{l}\text { DESARROLLO DE } \\
\text { HARWARE Y SU } \\
\text { SOFTWARE }\end{array}$ & $\begin{array}{l}\text { ADAPTACION DE } \\
\text { HARDVIARE Y/O } \\
\text { SOFTWARE PARA }\end{array}$ & $\begin{array}{l}\text { REDES DE } \\
\text { COOPUUTO } \\
\text { PARA }\end{array}$ \\
\hline & & & & $\begin{array}{l}\text { PARA } \\
\text { APLICACIONES } \\
\text { ESPECIFICAS }\end{array}$ & $\begin{array}{l}\text { IMPLEEENTACION } \\
\text { DE SISTEMAS DE } \\
\text { COMPUTO }\end{array}$ & $\begin{array}{l}\text { ASOCIADO PARA } \\
\text { APLICACIONES } \\
\text { ESPECIFICAS }\end{array}$ & $\begin{array}{l}\text { SOFFWALE PRA } \\
\text { APLCACIONES } \\
\text { ESPECIFICAS }\end{array}$ & $\begin{array}{l}\text { NARRA } \\
\text { NEESSDADSS } \\
\text { ESPECIFICAS }\end{array}$ \\
\hline INGLES C1 & & 89.4 & 94 & 0.5 & 0.5 & 0 & 0 & 0 \\
\hline INGLESC2 & & 77.9 & 81 & 0.5 & 0.5 & 0 & 0 & 0 \\
\hline ALGEBRA SUPERIOR & & 95.8 & 82 & 0.5 & 0.5 & 0 & 0 & 0 \\
\hline
\end{tabular}

$\cdots$

\begin{tabular}{|c|c|c|c|c|c|c|c|}
\hline ECUACIONES DIFERENCIALES & 93.9 & 75 & 0.5 & 0.5 & 0 & 0 & 0 \\
\hline CALCULO 2 & 87.7 & 67 & 0.5 & 0.5 & 0 & 0 & 0 \\
\hline \multirow[t]{6}{*}{ CALCULO 3} & 96.4 & 67 & 0.5 & 0.5 & 0 & 0 & 0 \\
\hline & \multicolumn{2}{|c|}{ Pertenencia Obtenida por Área (POA): } & 12.72 & 14.09 & (14.58) & 13.6 & 8.9 \\
\hline & \multirow{2}{*}{\multicolumn{2}{|c|}{ Estimación Simple: }} & 87.2428 & 96.6392 & 100.0 & 93.2785 & 61.0425 \\
\hline & & & 1117.36 & 1165.7 & 1196.49 & 1163.72 & 1018.74 \\
\hline & \multirow{2}{*}{\multicolumn{2}{|c|}{$\begin{array}{l}\text { Estimación por Periodo de Egreso: } \\
\text { Estimación por Años de Experiencia: }\end{array}$}} & 1004.62 & 1048.13 & 1075.84 & 1046.35 & 915.86 \\
\hline & & & 1159.635 & 1209.785 & 1241.725 & 1207.735 & 1057.315 \\
\hline
\end{tabular}

Fig. 7. Estimaciones del EGEL para Ingeniería en Computación

Por tanto, es aquí donde pudieran entrar las estrategias a seguir para fortalecer las áreas más desprotegidas. No obstante, para este sustentante el TD pronosticado del EGEL al que el mismo aspiraría es un TDS, el cual corresponde exactamente al TD real. Con esto, realizando un comparativo de la resta de los puntajes reales menos los puntajes del pronóstico se puede obtener la ecuación siguiente:

$$
\mathrm{CA}=\text { puntaje obtenido }-\mathrm{PAE}
$$

Donde CA: es el comparativo por área de los puntajes reales con los puntajes del pronóstico de cada área.

CA SSCAE $=-55.07$

CA NTISC $=16.87$

CA DHSAAE $=-76.63$

CA AHSAE $=-68.22$

$\mathrm{CA}$ RCNE $=24.70$ 
Lo anterior dice, que el pronóstico dado puede alejarse o acercarse en promedio en un valor de \pm 31.67 , lo cual quiere decir que en cada área el resultado pronosticado puede beneficiar o perjudicar al sustentante en dicho valor. No obstante, se debe contemplar además que por cada área dicho valor puede ser muy diferente.

Calculando el porcentaje del pronóstico de cada área se puede dar la siguiente ecuación:

$\mathrm{PPA}=100-($ valor absoluto $(100(\mathrm{PAE} * 100) /$ valor obtenido por área $))$

Donde PPA: es el porcentaje del pronóstico del área.

Los valores de los porcentajes del pronóstico obtenidos son:

PPA SSCAE $=94.69$

PPA NTISC $=98.51$

PPA DHSAAE $=92.83$

PPA AHSAE $=93.48$

PPA RCNE $=97.53$

Finalmente, los valores anteriores dicen que la aproximación del pronóstico generado es considerablemente acertada, ya que en promedio se tuvo un porcentaje de acierto del 95.40.

\section{Conclusiones}

El análisis y creación del modelo de pronóstico difuso aquí propuesto para el TD del EGEL de los sustentantes de ICO de la UAEM que opten por dicha modalidad de titulación, ha permitido detectar principalmente los siguientes aspectos a considerar:

1. El objetivo es acreditar el EGEL y no necesariamente medir el nivel de conocimiento adquirido.

2. Existen deficiencias notorias en el actual PE, esto va dado con respecto a las áreas que evalúa el EGEL.

3. El porcentaje de UDAs que impactan en el área de RNCE es bajo, por lo que, difícilmente un sustentante logrará alcanzar un TDS, a menos que éste, tenga experiencia en dicha área.

4. No se esperaría que exista un gran porcentaje de resultados reales con un TDSS.

Por lo anterior, si se desea que el EGEL sea una modalidad que cumpla con la meta establecida en el plan rector de la UAEM y que los egresados sean capaces de utilizar lo que han estudiado y aprendido en su licenciatura en situaciones similares a las que se enfrentarán en el ejercicio profesional, se requiere que se establezcan las siguientes medidas:

- Revisar la afinidad de contenidos de las UDAs del PE de ICO, con los reactivos que se aplican en las áreas del EGEL, a fin de adecuar y mejorar los contenidos temáticos y con ello se logre un mejor aprovechamiento de los alumnos. Por ende, un TDS es al menos el rango de puntuación deseado para aquellos que opten por el EGEL, un TDSS sería una ganancia adicional. 
- Estructurar un programa intensivo de preparación para los sustentantes que vayan a presentar el EGEL, considerando las áreas de menor fortaleza en la evaluación sin descuidar aquellas con mayor presencia.

\section{Referencias}

1. SEP: Lineamientos para la formulación de indicadores educativos. Disponible en http://fs.planeacion.sep.gob.mx/estadistica_e_indicadores/lineamientos_formulacion_de_i ndicadores.pdf, recuperado en marzo 2015 (2014).

2. ANUIES: Anuario estadístico de educación superior. Disponible en http://www.anuies.mx/iinformacion-y-servicios/informacion-estadistica-de-educacionsuperior/anuario-estadistico-de-educacion-superior, recuperado en marzo 2015 (20122013)

3. UAEM: Agenda Estadística 2012-2013. Disponible en http://www.uaemex.mx/planeacion/Numeros.html, recuperado en marzo 2015

4. UAEM: Reglamento de evaluación profesional. Disponible en http://www.uaemex.mx/opcevl/, recuperado en marzo 2015

5. CENEVAL: Guía para el sustentante, EGEL para Ingeniería Computacional. Disponible en http://archivos.ceneval.edu.mx/archivos_portal/18791/GuiadelEGEL-ICOMPU.pdf, recuperado en marzo 2015

6. Ponce, P.: Inteligencia artificial, con aplicaciones a la ingeniería. pp. 20-50, Alfaomega, México (2010)

7. Kumar, R.: Soft Computing and its Applications. vol. 1, pp. 213-287, Apple Academic Press (2015)

8. Chen, G., Pham, T., Introduction to Fuzzy Sets, Fuzzy Logic, and Fuzzy Control Systems. CRC Press (2000)

9. Cingolani, P., Alcala-Fdez, J.: jFuzzyLogic: a robust and flexible Fuzzy-Logic inference system language implementation. In: Fuzzy Systems (FUZZ-IEEE), 2012 IEEE International Conference on, IEEE, pp. 1-8 (2013) 